\title{
Segmented Terahertz device for ultrashort electron acceleration, compression, focusing and streaking
}

\author{
Dongfang Zhang ${ }^{1,2,{ }^{*}}$, Arya Fallahi $^{1}$, Michael Hemmer ${ }^{1}$, Xiaojun $\mathrm{Wu}^{1}$, Moein Fakhari $^{1,2}, Y i$ \\ Hua $^{1}$, Huseyin Cankaya ${ }^{1}$, Anne-Laure Calendron ${ }^{1}$, Luis E. Zapata ${ }^{1}$, Nicholas H. Matlis ${ }^{1}$ and \\ Franz X. Kärtner ${ }^{1,2,3}$ \\ ${ }^{1}$ Center for Free-Electron Laser Science, Deutsches Elektronen Synchrotron, Notkestrasse 85, 22607 \\ Hamburg, Germany. \\ ${ }^{2}$ Department of Physics and The Hamburg Centre for Ultrafast Imaging, University of Hamburg, \\ Luruper Chaussee 149, 22761 Hamburg, Germany. \\ ${ }^{3}$ Research Laboratory of Electronics, MIT, Cambridge, 02139 Massachusetts, USA.
}

\begin{abstract}
We present a segmented THz based device (STEAM) capable of performing multiple high-field operations on the 6D-phase-space of ultrashort electron bunches. With this single device, powered by fewmicro-Joule, single-cycle, $0.3 \mathrm{THz}$ pulses, we demonstrate record $\mathrm{THz}$ acceleration of $>30 \mathrm{keV}$, streaking with $<10$ fs resolution, focusing with $>2$ $\mathrm{kT} / \mathrm{m}$ strength, compression to $\sim 100$ fs. The STEAM device can be fabricated with regular mechanical machining tools and supports real-time switching between different modes of operation. It paves the way for the development of THz-based compact electron guns, accelerators, ultrafast electron diffractometers and Free-Electron Lasers.
\end{abstract}

Ultrafast electron beams are the basis for free-electron lasers, ultrafast streak cameras, and femtosecond electron diffractometers. Microwave technology at gigahertz frequencies have been the conventional choice with high degree of technical maturity and have been used extensively across all areas of industry and science. However, it has costly infrastructures and suffers from inherent difficulties in laser-electron synchronization that limits its further development for pursuing even higher temporal resolution. The electric fields governing emittance and bunch length are limited to $100 \mathrm{MV} / \mathrm{m}$. Strong motivation thus exists for exploring alternative technologies such as dielectric laser accelerators (DLAs) [1], laserplasma accelerators (LPAs) [2] and THz-driven accelerators [3], which provide intrinsic optical synchronization and substantially stronger fields. However, LPAs still suffer from instabilities and difficulties in controlling injection. DLAs, which employ micron-scale structures, require extreme tolerances on alignment and control, and are limited to sub-fC bunch charge. THz-based accelerators in the intermediate millimeter scale allow traditional fabrication techniques and support moderate charge $(\mathrm{pC})$ while still benefit from compactness, low cost, strong driving fields and field-gradients. This balance makes THz-

* Corresponding author: dongfang.zhang@cfel.de 
based acceleration a very promising technology for future devices. Here, we demonstrate a segmented THz waveguide device (STEAM device) that is capable of performing multiple high-field operations on the 6D-phase-space of ultrashort electron bunches [4].

The experimental setup (Fig.1) consisted of a $55 \mathrm{keV}$ photo-triggered DC gun, a THzpowered STEAM device for electron manipulation and a diagnostic section. Ultraviolet pulses for photoemission were generated by two successive stages of second harmonic generation ( $\mathrm{SHG}$ ), while single-cycle $\mathrm{THz}$ pulses were generated by difference frequency generation [5] and coupled into the STEAM device transversely. According to the Lorentz force law, the electrons experience both the electric and magnetic fields of the THz pulses during interaction. The electric field is responsible for acceleration and deceleration, while the magnetic field induces transverse deflections.

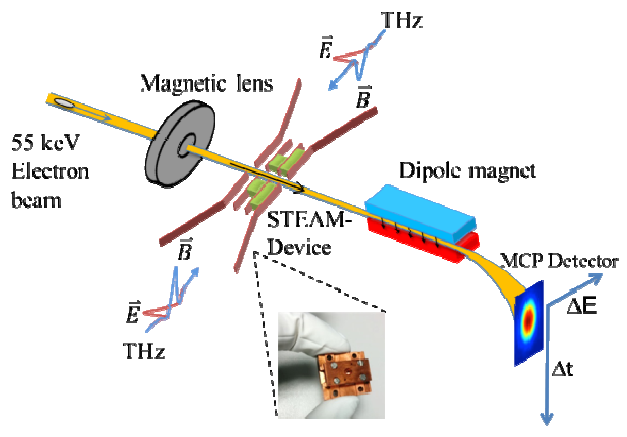

Fig. 1. Schematic illustration of the experimental setup. The two counter-propagating THz beams interact with the $55 \mathrm{keV}$ electron beam inside the STEAM device. Subsequently, the electron beam is detected by the camera. Inset: photograph of the STEAM device [4].

Efficient interaction of the electrons with the fields was accomplished by means of segmentation [6] which divides the THz-electron interaction volume into multiple layers of varying thickness (Fig. 1). Dielectric slabs of varying length were used to delay the arrival time of the $\mathrm{THz}$ waveform to coincide with the arrival of the electrons. The use of two counter-propagating drive pulses enabled two key modes of operation: an "electric" mode, used for acceleration, compression \& focusing, in which the fields were timed to produce electric-field superposition and magnetic-field cancellation at the center of the interaction point; a "magnetic" mode, used for deflection and streaking, where the magnetic fields superposed and the electric fields cancelled at the center of the interaction point.

The function of the device was thus selected by tuning the relative delay of the two $\mathrm{THz}$ pulses and the electrons. In the electric mode, maximum acceleration and deceleration occurred at the electron injection points. The peak field reached $\sim 70 \mathrm{MV} / \mathrm{m}$ resulting in more than $30 \mathrm{keV}$ acceleration (Fig. 2(a)) with the available $\sim 2 \times 6 \mu \mathrm{J}$ of coupled $\mathrm{THz}$ energy. At the zero-crossing of the field, the electric field imparts a temporally energy gradient resulting in a velocity gradient that causes either compression or stretching of the electron bunch as it propagates. Figure 2(b) shows the electron bunch temporal profiles measured at with a STEAM streaker for various field strengths applied to the STEAM buncher. A minimum duration of $100 \mathrm{fs}$ FWHM was achieved (Fig. 2(b)). At the same zero crossing, the STEAM device can also operate as a transverse focusing or defocusing element in parallel to the longitudinal decompressing and compressing fields, respectively. Figure 2(c) shows that at best focus the electron beam diameter was reduced by $2 \times$ compared to its input value. Peak focusing gradients reach over $2 \mathrm{kT} / \mathrm{m}$ that is close to the performance of active plasma lenses. 
In the magnetic mode, the relative timing of the $\mathrm{THz}$ fields is different from that of the electric mode by a half period, resulting in reinforcement of the magnetic field and cancellation of the electric fields at the center of the interaction region. When electrons are on crest of the magnetic field, the deflection is maximized. The electron beams can be precisely steered by varying the $\mathrm{THz}$ pulse energy. Electrons sweeping the zero crossing cycle of the $\mathrm{THz}$ magnetic field experience a deflection that is a steep function of time enabling to map out the temporal profiles of electron bunches by projecting them onto a spatial domain (Fig. 2(d)). For THz energies of $\sim 2 \times 6 \mu \mathrm{J}$, a maximum deflection gradient of $>140 \mu \mathrm{rad} / \mathrm{fs}$ was achieved. This corresponds to a temporal resolution below $10 \mathrm{fs}$.

(a)
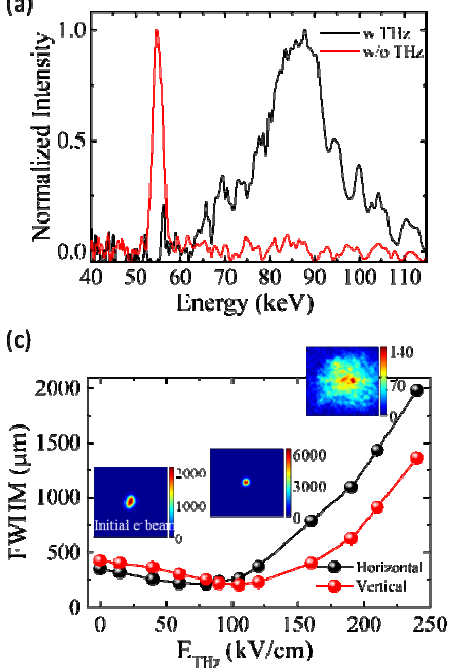

(b)

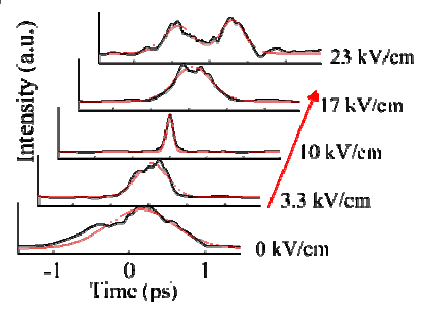

(d)

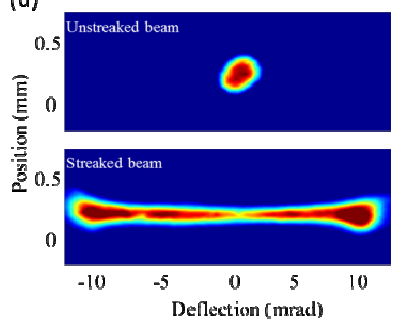

Fig. 2. (a) Electron energy spectra for input beam (red curve) and accelerated beam (black curve). (b) Temporal profiles of the electron pulses as the THz field in the buncher is increased. (c) Transverse electron beam size as a function of the $\mathrm{THz}$ field at the focusing mode. (d) Images of the electron beam on the detector with and without the THz deflection field [4].

In summary, we have demonstrated a novel segmented $\mathrm{THz}$ electron accelerator and manipulator setting new records in $\mathrm{THz}$ acceleration, streaking and focusing with a very compact device. The STEAM device demonstrates the feasibility of THz-based compact electron guns, accelerators, ultrafast electron diffractometers and Free-Electron Lasers with transformative impact.

[1] J. Breuer, P. Hommelhoff, Phys. Rev. Lett. 111, 134803 (2013).

[2] V. Malka, J. Faure, Y. A. Gauduel, E. Lefebvre, A. Rousse, K. T. Phuoc, Nature Physics 4, 447 (2008).

[3] E. A. Nanni, W. R. Huang, K. -H. Hong, K. Ravi, A. Fallahi, G. Moriena, R. J. D Miller, F. X. Kärtner, Nat. Commun 6, 8486 (2015).

[4] D. Zhang, A. Fallahi, M. Hemmer, X. Wu, M. Fakhari, Y. Hua, H. Cankaya, A.-L. Calendron, L. E. Zapata, N. H. Matlis, and F. X. Kärtner, Nat. Photonics 12, 336 (2018).

[5] J. Hebling, G. Almasi, I. Z. Kozma, J. Kuhl, Opt. Express 10, 1161 (2002).

[6] A. Fallahi, M. Fakhari, A. Yahaghi, M. Arrieta, F. X. Kärtner, Phys. Rev. Accel. Beams 19, 081302 (2016). 